\section{Original Article}

Dr. Md. Zahid Hossain BDS, PGD, PhD

Assoc. Professor \& Chairman

Division of Periodontics

Dept. of Preventive Dental Sciences College of Dentistry, King Khalid University Kingdom of Saudi Arabia

Dr. Hammam I. Fageeh

BDS

Intern Doctor

Division of Periodontics

Dept. of Preventive Dental Sciences College of Dentistry, King Khalid University Kingdom of Saudi Arabia.

Dr. Fadul A. Gader BDS, MSc

Asst. Professor

Division of Periodontics Dept. of Preventive Dental Sciences College of Dentistry, King Khalid University Kingdom of Saudi Arabia

Dr. M. Yunis Saleem BDS, MDS Assoc. Professor Division of Periodontics Dept. of Preventive Dental Sciences College of Dentistry, King Khalid University Kingdom of Saudi Arabia

Correspondence to : Dr. Md. Zahid Hossain BDS, PGD \& PhD (Japan)

Professor

Dept. of Periodontology, Oral Pathology \& Oral Medicine City Dental College, Dhaka e-mail: hoszahid@hotmail.com

\title{
Relationship of Blood Glucose Level and Severity of Periodontitis in Patients attended the Outpatient Periodontics Clinic of College of Dentistry, King Khalid University, Saudi Arabia
}

\begin{abstract}
:
Aims: A prospective cross-sectional study was performed involving 30 patients who attended the College of Dentistry outpatient Periodontics clinic of King Khalid University, Abha, Kingdom of Saudi Arabia during the period of 12th March -12th May, 2011.

Methods: Clinical data were recorded from the randomly selected 30 patients where periodontitis was indexed after Loe $\mathrm{H}$ : J Periodontol 38 (suppl):610, 1967 and modified from Russel AL: J Dent Res 35:350, 1956. Diabetic conditions were determined by 2 separate fasting plasma glucose (FPG) levels determinations [100 125 mg/dL at 8 hours fasting] and oral glucose tolerance test (OGTT) [140 199 $\mathrm{mg} / \mathrm{dL}$ at 2 hours after $75-\mathrm{g}$ glucose load].
\end{abstract}

Results: Among 30 patients, mean age 33 \pm 7 (range 18 54) years, males $70 \%$ and females $30 \%$ were divided into three groups: i) 10 periodontitis patients having diabetes mellitus (DM), ii) 10 non-diabetic periodontitis patients and iii) 10 patients were not suffering from periodontitis or DM as a control group. Results revealed that patients having diabetes had more moderate to severe forms of periodontitis $50 \%(p<0.05)$ including type $2(80 \%)$ than type1 $(20 \%) \mathrm{DM},(\mathrm{p}<0.05)$. Similarly, $80 \%$ of the periodontitis patients were found suffering from moderate to severe forms of periodontitis $(p<0.05)$ in uncontrolled group (20\%) of DM patients.

Conclusion: The present study proved a clear relationship with the patients having diabetes and periodontitis including severity of the diseases and glycemic control. Using these results, a greater effort can be made in providing periodontal as well as overall health to the population of at or around of Abha city of Saudi Arabia.

Key words: Blood glucose levels, periodontitis.

Introduction :

Periodontitis is defined as having at least one periodontal site with 3 millimeters or more of attachment loss and 4 millimeters or more of pocket depth. Using the American Academy of Periodontology (AAP) definition of moderate and severe periodontitis: Moderate periodontitis is defined as having at least two teeth with interproximal attachment loss of 4 millimeters or more or at least two teeth with 5 millimeters or more of pocket depth at interproximal sites. Severe periodontitis is defined as having at least 2 teeth with interproximal attachment loss of 6 millimeters or more and at least one tooth with 5 millimeters or more of pocket depth at interproximal sites. $^{1}$ 
Using data from the 1999-2004 National Health and Nutrition Examination Survey (NHANES), United States of America (USA) ${ }^{1}$, researchers demonstrated that adults with low income and less than a high school education are approximately twice as likely to have periodontal disease compared with more affluent adults with higher educational attainment. ${ }^{2}$ Physicians should be concerned about the high rates of tooth loss, and periodontal disease because these oral conditions are associated with significant negative health outcomes. Periodontal diseases have potential effects on a wide range of organ systems, e.g., coronary heart disease (CHD), atherosclerosis, myocardial /cerebral infarction, stroke, diabetes mellitus, preterm labor /low birth-weight baby, chronic obstructive pulmonary disease, acute respiratory infections and so on. ${ }^{1}$

Diabetes mellitus (DM) is characterized by increased levels of glucose in the blood and abnormalities in the metabolism of lipid and protein induced by diminished levels or total absence of insulin. Of all the best known systemic diseases, diabetes has been the one most frequently blamed as a risk agent for periodontal disease and other oral pathologic disorders. ${ }^{2}$ The American Diabetes Association Expert Committee in 1997 and 1998 has revised the diagnostic criteria for diabetes and has implemented changes in the 1979 classification as follows ${ }^{3}$ :

i. Use of the terms type 1 and type 2 diabetes instead of insulin-dependent (IDDM) and noninsulin-dependent (NIDDM) to refer to the two major types of DM.

ii. Use of two separate fasting plasma glucose (FPG) levels determinations $(100 \sim 125 \mathrm{mg} / \mathrm{dL}$ at 8 hours fasting) and oral glucose tolerance test (OGTT) (140 199 mg/dL at 2 hours after 75-g glucose load).

iii. $\mathrm{HbA} 1 \mathrm{c}$ value is greater than $6.5 \%$ on 2 separate occasions.

Type 1 diabetes is generally seen in patients below age 40 while type 2 tends to occur after age 40.3 The most marked oral complications for both type 1 and 2 diabetes are seen in uncontrolled diabetic patients. Intraoral findings include, periodontal disease which is more severe and with a higher prevalence than that seen in the non-diabetics. ${ }^{3-5}$

It has been statistically proven that diabetes is one of the predisposing factors for the development of periodontal disease. Recurrent periodontal abscesses are also typical of diabetic patients.
The clinical manifestations of periodontal disease in adults and young diabetics are more severe than those observed in the non-diabetic population. Those with diabetes have a greater prevalence of attachment loss and bone loss than aged matched controls. Diabetics also have increased severity of periodontal destruction with subjects 15 to 34 years old having twice the amount of periodontal destruction as normal subjects. ${ }^{2,3}$

The etiology of diabetes seems to be a combination of intrinsic (genetic) and environmental factors to the degree that some authors think of diabetes as a series of diseases that have glucose intolerance in common. Genetics plays a role but it is not properly understood for type 1 while it plays a much greater role for type ${ }^{2}$. Autoimmunity as well as viral infections such as congenital rubella, hepatitis, mumps and cytomegalovirus have been reported to trigger the development of type 1 diabetes. ${ }^{2,8}$ Accurate evidence for the causative role of viral infections is inconclusive. Hyperthyroidism, hyperpituitarism, steroid medication as well as the destruction of pancreatic beta cells by surgery, cancer or inflammation can induce the development of diabetes in susceptible persons. Susceptibility for type 1 diabetes is determined by human leukocyte antigens (HLA) which are located on the surface of T lymphocytes. HLA are genetically controlled. Type 2 diabetes is not associated with destruction of beta cells of the pancreas but with resistance to insulin, altered insulin secretion and elevated liver glucose production. Studies in identical twins have shown that there is a $90 \%$ concordance for both twins to develop diabetes type 2 . The genes for type 2 diabetes have not yet been mapped. Obesity plays an important role in the development of diabetes type 2 and this is well demonstrated by the fact that the vast majority of young patients which develop type 2 diabetes are obese. Contributing causative factors for type 2, besides obesity, are sedentary life and older age. As stated before, maturity onset diabetes of the young (type 1 diabetes) is inherited as an autosomal dominant trait. Gestational diabetes mellitus (GDM) develops during pregnancy. ${ }^{3-6}$

\section{Research Hypothesis:}

Prevalence of DM is about $3-7 \%$ in the western countries. ${ }^{6}$ In Saudi Arabia, where modernization and industrialization are rapidly increasing, the incidence of DM appears to be on the rise. A high prevalence of DM has been noted in Saudi populations $(2.55$ and $5.32 \%$ among males and females, respectively). ${ }^{8}$ It is also known, and worth mentioning here, that the prevalence and clinical features of diabetes mellitus vary from one ethnic group to another. ${ }^{2,8}$ 
The conflict of the results referred to might, therefore, be more apparent than real, the variability being related to both genetic and environmental factors such as over nutrition. According to this hypothesis, the authors felt it important to investigate the relationship between diabetes mellitus and dental diseases in an Arabian community, that is, in a relatively stable ethnic group. ${ }^{8}$

Only two studies have been found (through internet) carried out to determine the prevalence and relationship of periodontitis in diabetic patients at King abdul Aziz University Hospital, Jeddah and King Saud University, College of Dentistry, Riyadh, KSA. ${ }^{9,10}$

This has been the first effort of the College of Dentistry, KKU to conduct a research study regarding periodontitis and diabetes relationship status among the dental patients attended the outpatient Periodontics clinic. Using the research results of periodontitis-diabetes relationship, a greater measure can be taken to create awareness of people in and around the Abha city of Saudi Arabia.

\section{Methods :}

It is designed as a prospective, cross-sectional study, clinical data were recorded from the patients attended the OPC, College of Dentistry, KKU where types of Periodontitis were indexed after Loe H: J Periodontol 38 (suppl): 610, 1967 and modified from Russel AL: J Dent Res 35:350, 1956. Diabetic conditions were determined by- i) 2 separate FPG levels determinations (100 125 mg/dL at 8 hours fasting) and OGTT (140 199 mg/dL at 2 hours after 75-g glucose load) and ii) 2 separate $\mathrm{HbA} 1 \mathrm{c}$ values is (> $6.5 \%)$.

Subjects (study groups): Patients were selected for this research study as follows:

Group-1: Ten (10) periodontitis patients having DM.

Group-2: Ten (10) non-diabetic periodontitis patients.

Group-3: Ten (10) patients not suffering from periodontitis or DM (control group).

\section{Inclusion criteria:}

1- Diagnosed type 1 or 2 diabetics.

2- Male IFemale.

3- Saudi Inon-Saudi.

4- Age ranging from 18-55 years old.

5- No known diagnosis of periodontitis before diabetes is established.

\section{Exclusion criteria:}

1. Those with compromised medical, intellectual, or psychological conditions.

2. Antibiotic taken within last 6 weeks.

Parameters used:

i. Periodontal Pocket Depth (PPD).

ii. Clinical Attachment Loss (CAL).

iii. Bone Loss (BL).

iv. All Orthopantomograph (OPG)s .

v. Wisdom teeth were excluded.

\section{Statistical analysis:}

Statistical analysis were performed with the IBM mainframe version of SAS (statistical analysis system) utilizing the chi-square distribution and the significance value was set at $<0.05$.

\section{Results :}

Total number of patients showed that both of the patients groups with $(10 \%)$ or without $(60 \%)$ having DM were suffering from periodontitis in the 18-30 years age group (Fig.1). This figure also revealed that with increasing age (31-60 years), patients were found in more numbers of suffering from periodontitis having diabetes.

Fig. 2 is showing that overwhelming majority $(80 \%)$ of the periodontitis patients were suffering from type 2 (non-insulin-dependent) DM and only $20 \%$ patients were suffering from type 1 (insulin-dependent) DM. Type 2 diabetic patients were suffering from all forms of periodontitis among whom most $(40 \%)$ of them were suffering from severe form of periodontitis. This results were significantly different $(p<0.05)$.

Age-wise distribution showed that among the nondiabetics, only $10 \%$ patients were suffering from severe form of periodontitis in 41-50 years age group (Fig.3), whereas, 18-30 years and 31-40 years age groups were suffering more from moderate $(40 \%$ and $10 \%$, respectively) and mild (20\% in each group) forms of periodontitis $(p<0.05)$. On the other side, in age-wise distribution among the diabetics, more (20\%) patients were suffering from severe form of periodontitis in 51-60 years age group (Fig.4). This figure also revealed that with increasing ages, severity of periodontitis were also increased $(p<0.05)$.

Among the diabetic patients, most $(80 \%)$ were suffering from moderate to severe forms of periodontitis in uncontrolled $(297 \pm 83 \mathrm{~g} / \mathrm{dL})$ DM group (Fig.5) $(p<0.05)$, whereas, rest $(20 \%)$ of patients were suffered from mild form of periodontitis in controlled $(105 \pm 10 \mathrm{~g} / \mathrm{dL})$ group of DM patients. 
Periodontitis patients with and without DM had significantly $(p<0.05)$ higher clinical attachment loss $(3.16 \pm 1.66 \mathrm{~mm}$ and $2.52 \pm 2.18 \mathrm{~mm}$, respectively) (Fig.6), mean bone loss $(33.78 \pm 22.61 \%$ and $27.82 \pm 19.04 \%$, respectively) and vertical bone loss $(37.79 \pm 21.73 \%$ and $26.43 \pm 16.5 \%$, respectively) (Fig.7). Whereas, horizontal bone loss was found more $(73.57 \pm 16.5 \%)$ in periodontitis patients without DM in comparison to the diabetic patients $(61.21 \pm 22.47 \%)$.

Fig.1: AGE-wise Distribution of Patients.

$(n=30)$.

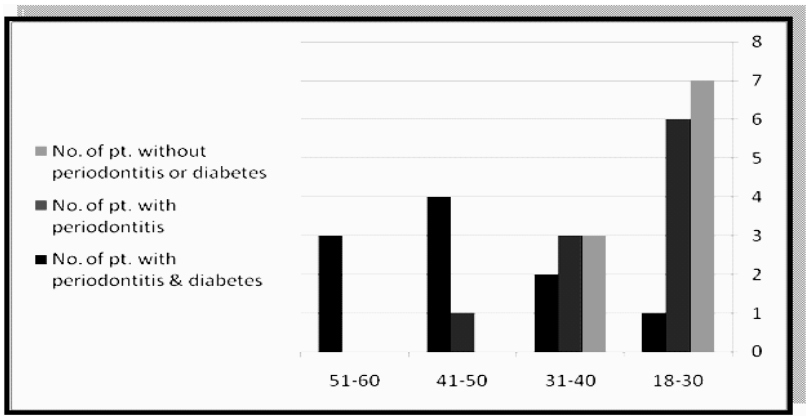

N.B.: $\mathrm{CP}(\mathrm{S})$ - chronic periodontitis mild type, $\mathrm{CP}(\mathrm{M})$ - chronic periodontitis moderate type and $\mathrm{CP}(\mathrm{A})$ - chronic periodontitis severe /advanced type. DM- diabetes mellitus. pt.- patients.

Fig.2: Severity of Periodontitis among patients with the types of DM.

$(n=10)$.

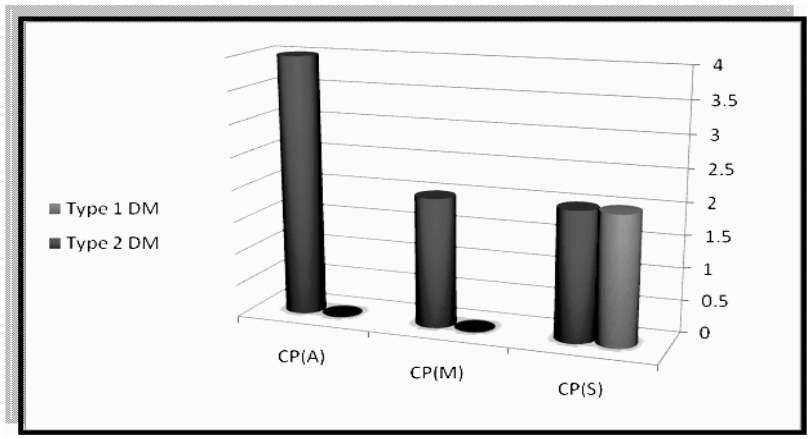

N.B.: $C P(S)$ - chronic periodontitis mild type, $C P(M)$ - chronic periodontitis moderate type and $\mathrm{CP}(\mathrm{A})$ - chronic periodontitis severe /advanced type. DM- diabetes mellitus.

Fig.3: Severity of periodontitis among patients without DM. $(n=10)$

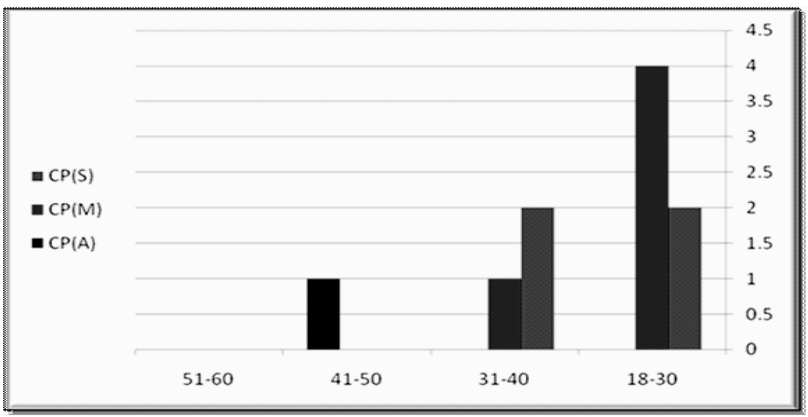

N.B.: $C P(S)$ - chronic periodontitis mild type, $C P(M)$ - chronic periodontitis moderate type and $\mathrm{CP}(\mathrm{A})$ - chronic periodontitis severe /advanced type. DM- diabetes mellitus.
Fig.4: Severity of Periodontitis among patients with DM. $\quad(n=10)$.

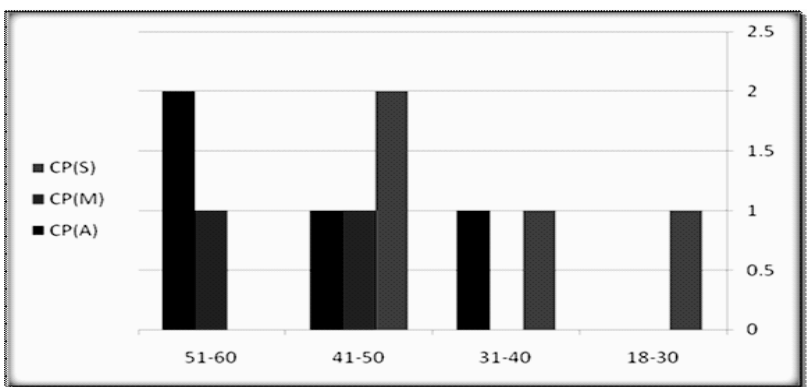

N.B.: $\mathrm{CP}(\mathrm{S})$ - chronic periodontitis mild type, $\mathrm{CP}(\mathrm{M})$ - chronic periodontitis moderate type and $\mathrm{CP}(\mathrm{A})$ - chronic periodontitis sever /advanced type. DM- diabetes mellitus.

Fig.5 : Severity of periodontitis patients with controlled $(105 \pm 10 \mathrm{mg} / \mathrm{dL})$ and uncontrolled $(297 \pm 83 \mathrm{mg} / \mathrm{dL})$ DM. $(\mathrm{n}=10)$.

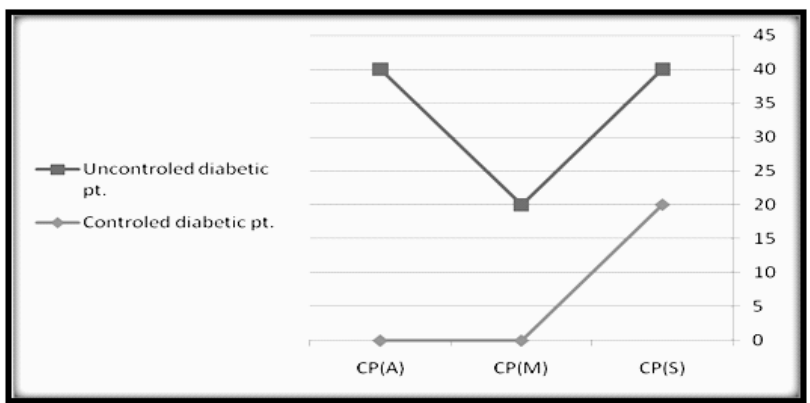

N.B.: $\mathrm{CP}(\mathrm{S})$ - chronic periodontitis mild type, $\mathrm{CP}(\mathrm{M})$ - chronic periodontitis moderate type and $\mathrm{CP}(\mathrm{A})$ - chronic periodontitis severe /advanced type. DM- diabetes mellitus. pt.- patients.

Fig.6 : Severity of clinical attachment loss (CAL) in periodontitis patients with or without DM. $\quad(n=10)$.

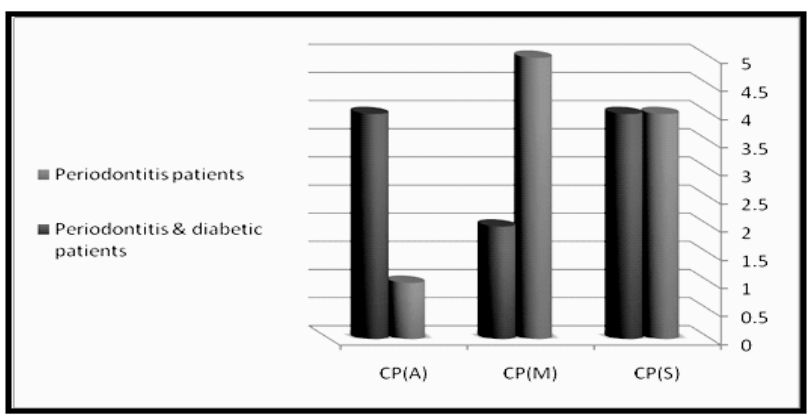

N.B.: $\mathrm{CP}(\mathrm{S})$ - chronic periodontitis mild type, $\mathrm{CP}(\mathrm{M})$ - chronic periodontitis moderate type and $\mathrm{CP}(\mathrm{A})$ - chronic periodontitis severe /advanced type. DM- diabetes mellitus.

Fig.7 : Mean, horizontal and vertical bone losses of periodontitis patients with or without DM.

$(n=10)$.

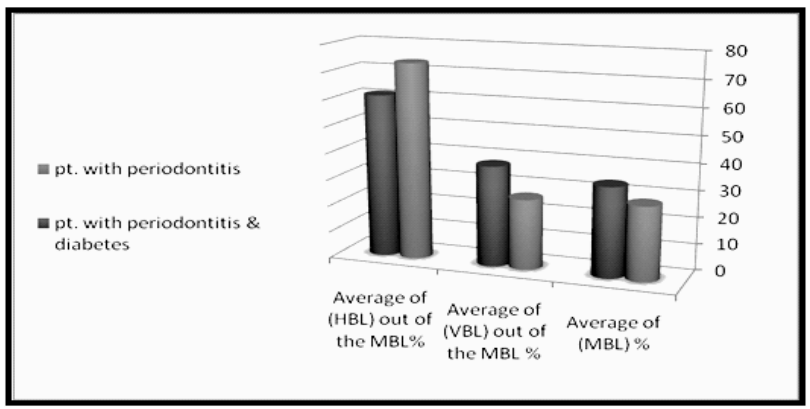

N.B.: pt.- patients, HBL- horizontal bone loss, VBL- vertical bone loss and MBL- mean bone loss. 


\section{Discussion :}

It has been statistically proven that diabetes is one of the predisposing factors for the development of periodontal disease $\mathrm{e}^{2,3}$ which is similar to the findings that is shown in Fig.1 The most marked oral complications for both type 1 and 2 diabetes are seen in uncontrolled diabetic patients and periodontal disease is more severe and with a higher prevalence than that seen in the non-diabetics. ${ }^{3}$ This study has supported these comments that $80 \% \quad(p<0.05)$ of periodontitis patients had type 2 and uncontrolled diabetic patients (Fig.2 \& 5).

The clinical manifestations of periodontal disease in adults and young diabetics are more severe than those observed in the non-diabetic population. ${ }^{2,3}$ In this study, data revealed that significantly $(p<0.05)$ more patients were found suffering from moderate to advanced types of periodontitis in the older age groups i.e., 31-60 years (Fig.3 \& 4).

Fig.6 \& 7 showed a greater prevalence of average clinical attachment loss (CAL) and bone loss (MBL\%) in aged than that of younger group of either diabetic or non-diabetic patients. Thus agreed with the statement that diabetic patients have a greater prevalence of attachment loss (CAL) and bone loss $(\mathrm{BL})$ than aged matched controls. ${ }^{2,3}$ This study also explored that higher CAL (data not shown) and angular /vertical $\mathrm{BL}$ were more in percentages than that of patients having diabetes (Fig.6) which is a sign of more devastating periodontal disease.

The present study was an approach of periodontal research on a small numbers of subjects at a short period of working time. Under such limitations, the research study was performed in proper ways of collecting data and data analysis.

Conclusions :

A large number of people at and around Abha city have been suffering from diabetes (data not shown) and diabetes have a great impact of periodontal status of those people and therefore, loss of teeth affecting their overall health.Thus the results concluded that the present study proved a clear relationship with the patients having diabetes and periodontitis including severity of the diseases and glycemic control. Using these results, a greater effort can be made in providing periodontal as well as overall health to the population of at or around of Abha city of Kingdom of Saudi Arabia. Further broad scale studies will be needed to have a concrete conclusion of the said topic.

\section{Acknowledgment :}

The Authors express their gratitude and acknowledgement to Dr. Nazar Omer Abdallah, Asst. Professor, Dept. of Business Administration, College of Community, KKU for his cordial assistance in analyzing the data.

\section{References :}

1. Committee on Research, Science, and Therapy of the American Academy of Periodontology: position paper: Academy report on epidemiology of periodontal diseases. J Periodontol 2005;76:1406-1419.

2. Tervonen T, Karjalainen K. Periodontal disease related to diabetic status. A pilot study of the response to periodontal therapy in type 1 diabetes. J Clin Periodont 1997;24:505-10.

3. Alberti KG, Zimmet PZ. Definition, diagnosis and classification of diabetes mellitus and its complications. Part 1: diagnosis and classification of diabetes mellitus provisional report of a WHO consultation. Diab Med 1998;15:539-53.

4. Committee on Research, Science, and Therapy of the American Academy of Periodontology: position paper: diabetes and periodontal disease. J Periodontol 1996;67:166-176.

5. Loe H. Periodontal disease: the sixth complication of diabetes mellitus. Diabetes Care 1993;16:329-334.

6. Eckman MH, Greenfield S, Mackey WC, et al. Foot infections in diabetic patients. Decision and costeffectiveness analyses. JAMA 1995;273(9):712-20.

7. Karim A, Ogbeide DO, Siddiqui S, et al. Prevalence of diabetes mellitus in a Saudi community. Saudi Med J. 2000;21(5):438-42.

8. Rimoin DL, Schimke RN. Genetic disorders of the endocrine glands. St. Louis:CV Mosby Co; 1971:150216.

9. Almas K, Al-Lazzam S, Al-Quadairi A. The effect of oral hygiene instructions on diabetic type 2 male patients with periodontal diseases. J Contemp Dent Pract 2003;(4)3:40-51

10. Al-Khateeb TL, Al-Amoudi $\mathrm{NH}$, Fatani $\mathrm{HH}$, et al. Periodontal diseases and caries experience of diabetic patients in an Arabian community. The Saudi Dent $J$ 1990;2(3):91-95. 